(C) 1998 IEEE. Personal use of this material is permitted. Permission from IEEE must be obtained for all other uses, in any current or future media, including reprinting/republishing this material for advertising or promotional purposes, creating new collective works, for resale or redistribution to servers or lists, or reuse of any copyrighted component of this work in other works. 


\title{
A Depth-Encoding PET Detector Module with Improved Spatial Sampling
}

\author{
J.J. Vaquero, J. Seidel, S. Siegel ${ }^{1}$, M.V. Green \\ Nuclear Medicine Department \\ National Institutes of Health, Bethesda MD 20892
}

\begin{abstract}
Detector modules in small ring diameter PET scanners must possess depth-of-interaction (DOI) encoding, increased spatial sampling, high sensitivity and the ability to handle high photon input rates without excessive pulse pileup or random coincidences. We created such a module by optically coupling an entrance array of individual LGSO crystals to an exit array of individual GSO (and other) crystals that was, in turn, optically and directly coupled to a miniature PSPMT. DOI was determined for each event by delayed charge integration (DCI), a technique that exploits differences in light decay time between GSO and LGSO.

Spatial sampling in 3D was increased by introducing a half crystal pitch spatial offset between the entrance and exit arrays in both the $\mathrm{X}$ and $\mathrm{Y}$ directions. Position detection accuracy in both the LGSO and GSO layers, and the accuracy of DOI assignment of events to either layer was high. These results suggest that this combination of scintillators and acquisition/processing methods may be particularly useful in the design of high performance, small ring diameter PET scanners for small animal imaging.
\end{abstract}

\section{INTRODUCTION}

As the diameter of a PET ring scanner shrinks, the need for depth-of-interaction (DOI) information, finer spatial sampling and improved count rate performance increases. Numerous methods have, in fact, been advanced that address one or more of these issues. We propose here a module that, in principle, meets all or most of these conditions simultaneously, while possessing good position detection accuracy.

The module combines five different features. First, the module is comprised of two layers of optically coupled scintillators that differ from each other in light decay time (for DOI determination). Second, all of the crystals in both layers are geometrically identical and possess small entrance crosssections (potentially good position detection accuracy). Third, the entrance layer is spatially offset with respect to the exit layer by half a crystal pitch in both spatial directions (increased spatial sampling in 3D). Fourth, both scintillators have short light decay times, high densities, good light outputs and an extended combined length (potentially high count rates with low pulse pileup, good stopping power, good position detection accuracy, good sensitivity).

Finally, these layers are directly coupled to a thin window, miniature position-sensitive photomultiplier tube with favorable performance characteristics (good timing properties

\footnotetext{
${ }^{1}$ Current address: Concorde Microsystems, 10427 Cogdill Rd, Suite 500, Knoxville, Tennessee 37932
}

for DOI discrimination, efficient conversion of scintillation light to photoelectrons for good position detection accuracy).

In the work described below, we evaluated a test module with these features for the purpose of determining whether this module might be suitable as the detection element of a high resolution, small diameter rodent PET scanner.

\section{MATERIALS AND METHODS}

\section{A. Detector Module}

The geometrical layout of the entrance and exit crystal arrays is shown in Figure 1. All crystals were $2 \mathrm{~mm} \times 2 \mathrm{~mm} x$ $10 \mathrm{~mm}$ long.
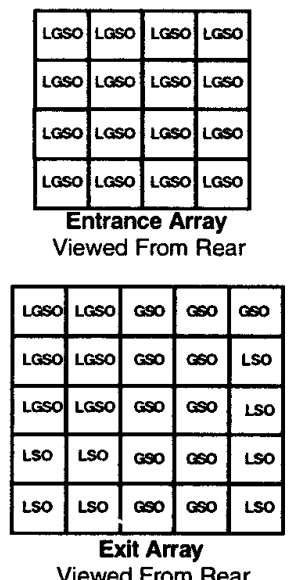

Figure 1. Geometrical layout of the crystal arrays. The entrance array is centered on, and coupled to, the exit array with a half pitch offset in both the $\mathrm{X}$ and $\mathrm{Y}$ directions.

All crystal surfaces were finished by chemical etching and all crystals were wrapped on their long sides with a double layer of Teflon tape. The entrance end of each crystal in the entrance layer was also covered with Teflon tape.

The entrance layers consisted only of LGSO crystals while the exit layer consisted of GSO and other scintillator types. (These other crystal types were included to evaluate different scintillator combinations and DOI methods, but here we shall focus only on the properties of the LGSO/GSO portion of the combined arrays).

Note that the entrance layer is offset by half a crystal pitch $(2.2 \mathrm{~mm} / 2=1.1 \mathrm{~mm})$ with respect to the exit array in both the $\mathrm{X}$ and $\mathrm{Y}$ directions. The entrance layer was optically coupled to the exit layer with optical grease and the exit layer coupled with optical grease to a Hamamatsu R5900-C8 positionsensitive photomultiplier tube (PSPMT). The entire assembly was placed in a light-tight box. 


\section{B. Data Acquisition/Processing}

For each scintillation event within its field-of-view, the PSPMT generates 9 signals, one for each of the four $\mathrm{X}$-anode plates, one for each of the four $\mathrm{Y}$-anode plates and a ninth signal from the last dynode for triggering and DOI discrimination (Figure 2). The dynode signal is first used to generate a $200 \mathrm{~ns}$-wide trigger signal for the chargeintegrating ADCs (LeCroy FERA). It is further split into two halves that are also integrated.

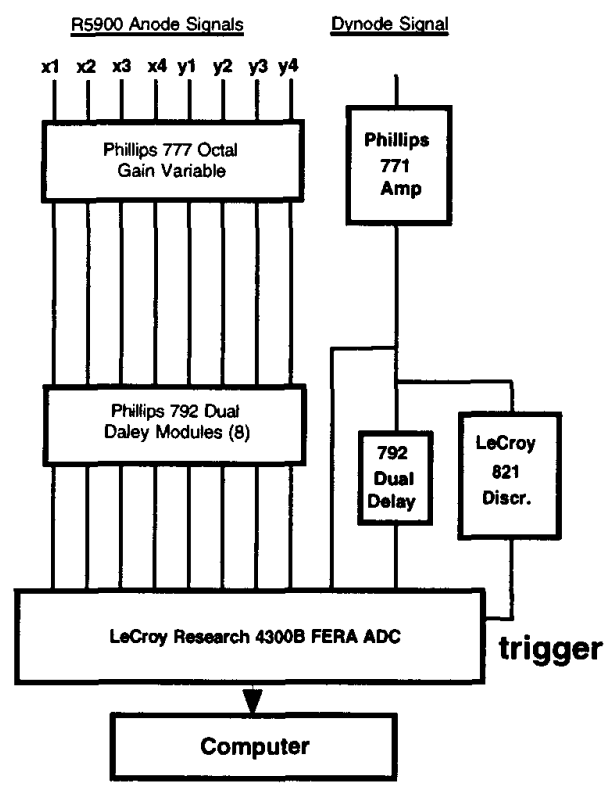

Figure 2. Data acquisition system.

All anode signals and one of the dynode signals are delayed for $100 \mathrm{~ns}$ to ensure that they arrive at the ADCs immediately after the trigger signal (Full Charge Integration, Figure 3). The other dynode signal is not delayed so that the ADC can only capture the charge that is still present after 100 ns (Delayed Charge Integration, Figure 3). If the full charge integral is plotted against the delayed charge integral (Figure $4 \mathrm{~A}$ ), two radial "spokes" appear in the resulting diagram, one for each scintillator.

Events occurring in the spoke with the greater slope correspond to events in the fast scintillators LGSO/LSO, while the spoke with the smaller slope corresponds to events in the slower GSO scintillator. If ROIs are defined that encompass the LGSO and GSO regions individually (Figure 4B), assignment of an event to one or the other of these regions also identifies the layer of interaction.

Thus, when an event occurs, depth is determined for that event by delayed charge integration (DCI) and the crystal of interaction in that layer determined by centroid calculation using the anode signals. (Subsequent refinement of the DCI technique has improved the separation between these two scintillators substantially as shown in Figure 4C).

\section{Experiments to Determine DOI and Event Positioning Accuracy}

The ability of these methods to place a scintillation event in the correct crystal were evaluated qualitatively by floodfield illumination of the module from the front. Because other scintillators were included in the exit array, the calculated pattern of events assigned to each layer can be compared to the known pattern of crystals in both layers.
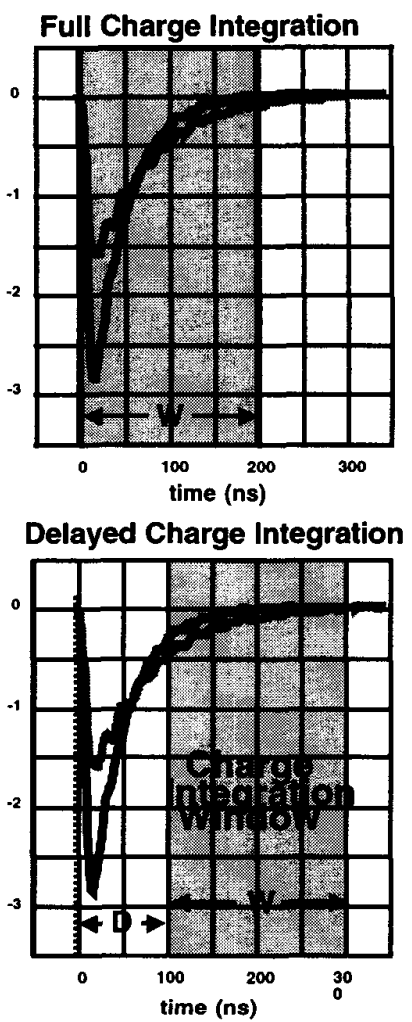

Figure 3. Illustration of the Delayed Charge Integration method.

DOI accuracy was determined by scanning a $1.1 \mathrm{~mm}$ wide F-18 slit source along the depth direction of the scintillator arrays. At each point, the number of events occurring in both scintillation layers was determined.

Similarly, position detection accuracy was determined for each layer by scanning a highly collimated beam of F-18 photons in $0.25 \mathrm{~mm}$ steps down the LGSO column in the entrance array that overlay the two columns of GSO crystals in the exit array. At each spatial position, the counts occurring in each crystal in both arrays were determined.

\section{RESULTS}

The results of field flood illumination of the module are shown in Figure 5. If the DOI selection conditions are not applied, an image of the field-of-view appears as in Figure 5A. When the DOI conditions are applied, however, this image separates into two images, one corresponding to the fast scintillators LGSO/LSO (Figure 5B) and the other to the slow scintillator GSO (Figure $5 \mathrm{C}$ ). Note that the pattern of GSO in Figure 5B and the combined pattern of LGSO/LSO crystals in 
the entrance and exit arrays shown in these figures correspond to the known pattern of crystals in the actual arrays (Figure 1).

DOI and position detection accuracy are illustrated in Figures 6 and 7. In each of these graphs the fraction of events assigned to each crystal is plotted against the position of the illuminating source. No additional event rejection conditions were applied in the creation of the DOI graph. However, spatial rejection conditions were applied before creating the position detection accuracy graph in Figure 7.

These results were obtained by placing small spatial ROIs around each crystal center and rejecting all events that occurred outside these regions. Under these conditions, position detection accuracy is high ( $>85 \%$ in both layers) but sensitivity low ( $<40 \%$ of all events are accepted). When large ROIs are used, position detection accuracy decreases $(<75 \%)$ but sensitivity is increased ( $>85 \%$ of all events are accepted).
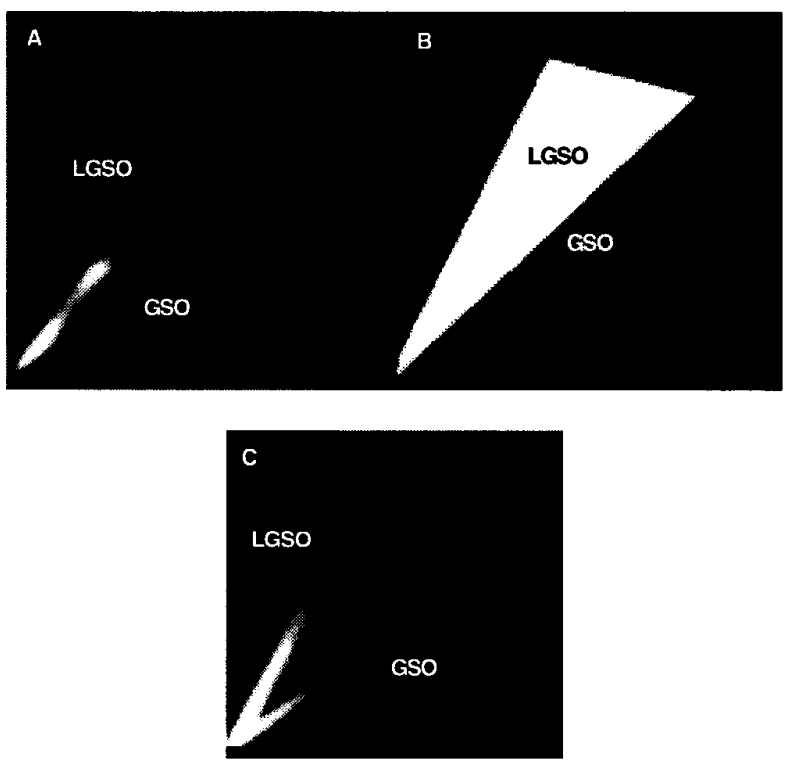

Figure 4. Phoswich diagram ( $\mathrm{A}$ and $\mathrm{C}$ ) created by plotting the full charge integral (vertical axis) against the delayed charge integral (horizontal axis). Definition of LUTs for DOI assigment (B). Separation is improved in (C) compared to (A) by reducing the dynode time constant.

\section{DISCUSSION AND CONCLUSION}

A phoswich/PSPMT detector module comprised of two fast, high stopping power scintillators with offset entrance and exit arrays can accurately locate the endpoints of annihilation events in space, albeit coarsely in the depth dimension. The spatial offset between entrance and exit arrays, moreover, does not appear to unduly degrade the accuracy of event positioning across the field-of-view. In addition, since both LGSO and GSO have short light decay times, pulse pileup can be minimized by utilizing short pulse integration times ( $200 \mathrm{~ns}$ or less).
The ability to acquire accurate DOI and position information at high rates and with relatively fine 3D spatial sampling suggests that this module may be useful in applications where these advantages are particularly significant, i.e. in small ring diameter PET scanners intended for small animal imaging.
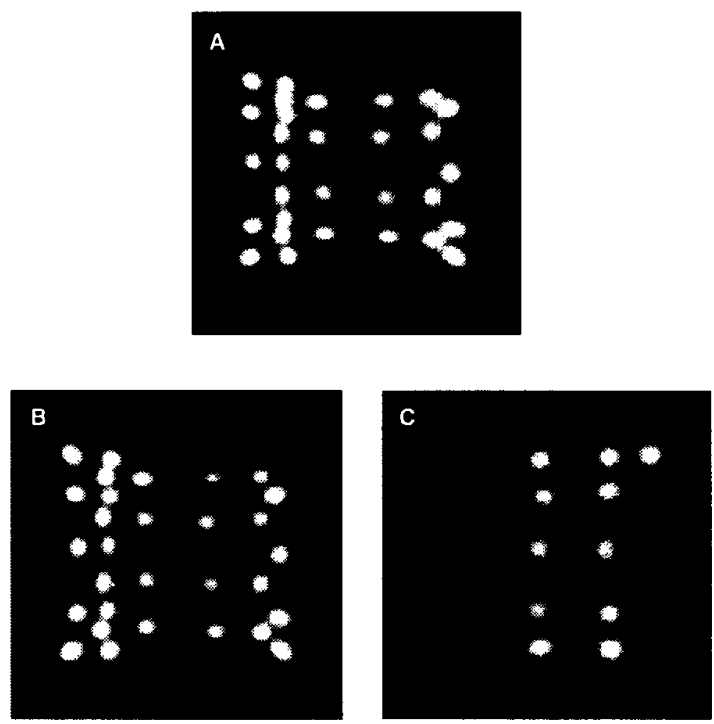

Figure 5. Field flood illumination of the detector module ignoring DOI information (A). This image separates into an LGSO/LSO image (B) and a GSO image (C) when the Delayed Charge Integration method is applied.

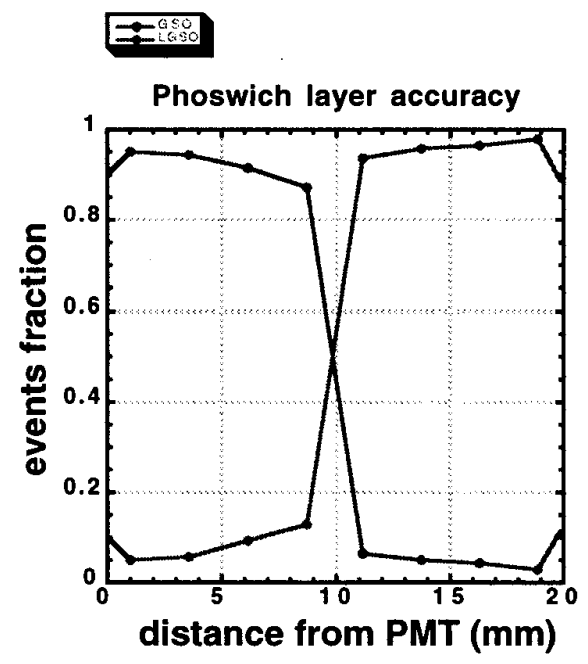

Figure 6. Fraction of events occurring in each phoswich layer when scanned by a narrow slit source. 

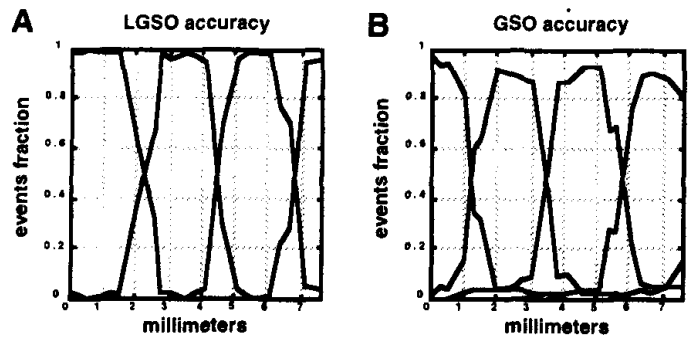

Figure 7. Fraction of events occurring in four adjacent LGSO crystals (A) and in four adjacent GSO crystals (B) when scanned by a narrow beam of $511 \mathrm{keV}$ photons.

\section{ACKNOWLEDGMENTS}

J.J. Vaquero is supported, in part, by a grant from CICYT (Spanish Government). S. Siegel was supported by a grant from the National Research Council.

\section{REFERENCES}

[1] M. Schmand, L. Eriksson, M.E. Casey, M.S. Andreaco, C. Melcher, K. Wienhard, G. Flagge, R. Nutt. "Performance Results of a New DOI Detector Block for a High Resolution PET-LSO Research Tomograph HRRT". 1997 IEEE Nuclear Science Symposium and Medical Imaging Conference Record.

[2] J.J. Vaquero, J. Seidel, S. Siegel, W.R. Gandler, M.V. Green. "Performance Characteristics of a Compact Position-Sensitive LSO Detector Module". IEEE Trans Med Imag, in press. 